\title{
Aportaciones a la construcción de un modelo de desarrollo local: La experiencia del Ayuntamiento de Catarroja
}

\author{
Ricard Calvo Palomares \\ Universidad de Valencia \\ Ricardo.Calvo@uv.edu \\ Juli Antoni Aguado i Hernàndez \\ Ayuntamiento de Catarroja \\ jaguado@catarroja.es
}

\begin{abstract}
Resumen
En la sociedad actual, la información se ha convertido en el intangible más preciado para cualquier organización que pretenda alcanzar sus objetivos en términos de eficiencia. Este artículo centra su interés en la importancia que tiene, para la administración pública local contemporánea, contar con información suficiente de sus actuaciones para mejorar su intervención sobre el territorio y en las dificultades que encuentra en la generación de conocimiento sobre los procesos de desarrollo socioeconómico. Frente a esta situación, se presentan las aportaciones al modelo de desarrollo local del sistema de información permanente de la realidad socioeconómica del Ayuntamiento de Catarroja (Valencia). Éste pone de manifiesto cómo las agencias de desarrollo local están en condiciones de convertirse en un observatorio del territorio en el cual actúan y en una de las principales fuentes de información para los agentes institucionales, económicos y sociales del mismo.
\end{abstract}

Palabras clave Intervención pública, desarrollo local, política pública, análisis de políticas públicas, Ayuntamiento de Catarroja.

\section{Contributions to the construction of a local development model: The experience of Catarroja City Council}

\begin{abstract}
In today's society, the information has become the most valuable intangible for any organization willing to achieve its objectives in terms of efficiency. This article focuses on how important is for contemporary public local administrations to have proper information of its actions in order to improve its intervention strategies on the territory. Moreover, the article includes conclusions about which are the main difficulties in generating knowledge about the processes of socio-economic development. Facing this scene, we present our contributions to the local development model of the permanent information system of the socio-economic reality of the City Council of Catarroja (Valencia, Spain). This model shows how local development agencies are able to become an observatory of the territory in which they act and one of the main sources of information for the institutional, economic and social agents.
\end{abstract}

Keywords

Public involvement, local development, public policy, public policy analysis, Catarroja City Council. 


\section{INTRODUCCIÓN}

En la actualidad, el alcance potencialmente ilimitado de la información deriva en incertidumbre; es decir, en la dificultad de establecer relaciones entre la cantidad de información que transmitimos y recibimos que, sin embargo, no garantiza su conversión en conocimiento porque aumenta el número de opciones posibles, pero también hace más difícil adoptar decisiones (Melucci, 1994: 132; Cornella, 2004: 1).

En este contexto de falta de certeza, donde la única posibilidad de mantener ventajas competitivas sostenibles por parte de los territorios, las administraciones, las economías y las empresas es innovar continuamente (Vázquez Barquero, 1999: 123; Boixader, 2007: 395), adquiere gran importancia la capacitación de estos para captar, procesar y utilizar productivamente la información y así apuntalarse en el conocimiento -en la certidumbre-, articulando los dos significados complementarios que expresa el término "información”. Éstos son: "informarse de" -extraer información, mediante la observación (semántica) - y “dar forma a” -inyectar neguentropía, mediante la acción (pragmática)- (Ibáñez, 1994: 2; Navarro, 1989: 43). Información "pragmática” que aporta instrucciones e indica cómo hacer las cosas, así como las posibilidades para concretarlas.

Por tal razón, los encargados de formular las políticas públicas deben ser capaces de generar unidades de observación de sus organizaciones, de su ámbito de actuación y de su entorno que busquen proactivamente información adecuada, cercana y útil, y la valoren y analicen mediante un conjunto de instrumentos específicos de aproximación y análisis de la realidad. Deben contar con investigaciones aplicadas procedentes de las ciencias sociales que sean pertinentes, oportunas y competentes metodológicamente -con el método, rigor y objetividad científicos-, por medio de la construcción de matrices o formatos para la estructuración de datos -como la que describimos en la experiencia de Catarroja (SDLPC, 2009; Aguado, 2010)- y la capacidad de la razón que aporta la ciencia para aprehender las tendencias existentes, comprender mejor y ser capaces de responder a las cuestiones fundamentales a las cuales se enfrentan (Morales, 1997: 1; Alós, 1999: 58; Angeleri, 1999: 15; Sanchis et al., 2001: 21; Asensi-Artiga y Parra-Pujante, 2002: 9-10, 12; Castro, 2002: 3, 192; Moltó y Hernández, 2002: 175, 178; UTEDLT, 2002: 2; Valdivielso, 2002: 24-25; Lamo de Espinosa, 2005; Wilson, 2005: I; León, Porras y Carmona, 2006: 6).

Un requisito de cualquier intervención pública es que existan unos objetivos determinados que se ajusten a las necesidades del territorio y que sean medibles sus

1 Atendiendo al modelo de análisis de las formas simbólicas -acciones, objetos o expresiones significantes, portadoras de información para los seres humanos- que desarrolla Ariño (1997: 82-83) y las modalidades fundamentales de interpelación ideológica de Therborn (1987: 15-16), puede considerarse que la "información pragmática" es la que aporta instrucciones y nos indica cómo hacer las cosas. No es verdadera ni falsa, sino eficaz o ineficaz en la consecución de resultados (Martín Criado, 1998: 70). Sobre la lengua como medio de, o como medio para, alcanzar algún fin por parte de los actores sociales véase Pérez-Agote y Tejerina (1990) y Tejerina (1992). 
resultados; por lo que, en el marco del análisis del proceso de las políticas públicas (Ferri, 2002; Valdivielso, 2002), la investigación social tiene un papel más o menos explícito en todas las fases a través de las cuales puede pasar un problema público, según la división de las etapas del proceso de las políticas públicas que desarrolla Valdivielso (2002). ${ }^{2}$

Desde el punto de vista del desarrollo local, política de intervención pública sobre la que pivota el presente artículo, hay que tener en cuenta que los fenómenos sociales y territoriales son extraordinariamente dinámicos tanto en el tiempo como en el espacio, por lo que su comprensión reclama un seguimiento de carácter constante (Melucci, 1994: 129-130; Castells, 1996: 47; Otero i Serra, 1998: 3; Sanchis et al., 2001: 23; León, Porras y Carmona, 2006: 3; Sevilla, 2006: 3).

Por ello, en primer lugar, la investigación social es necesaria para disponer de información territorial útil y de un tratamiento adecuado de la misma con indicadores cercanos y prácticos para identificar las oportunidades de intervención gubernamental y privada; por tanto, ésta puede ser una herramienta determinante en un proceso de toma de decisiones con intención de anticipación y basado en datos objetivos, mostrando los vínculos existentes entre la información socioeconómica y las tareas de gobierno (Morales, 1997: 1; Castro, 2002: 192, 245; Wilson, 2005: I; León, Porras y Carmona, 2006: 3, 6).

Asimismo, puede otorgar solidez al proceso de planificación de estas políticas, de forma objetiva y sustentable, teniendo en cuenta aspectos como el entorno y la cultura, la economía y el mercado de trabajo local, el medio ambiente y las infraestructuras, los recursos disponibles en la zona y las actividades de desarrollo en curso. Por ello, un conocimiento previo, más o menos detallado de estos aspectos, y adaptado a las características concretas de cada espacio, puede ser de gran utilidad para conocer el marco en el cual se procederá al desarrollo, disponiendo de bases reales para iniciar dicho proceso y, así, elaborar propuestas que permitan definir y ajustar políticas, estrategias de actuación y niveles de coordinación para mejorar resultados e impactos (Morales, 1997: 1; Sanchis et al., 2001: 21; Arriagada, 2002: 15; UTEDLT, 2002: 2; Wilson, 2005: I; León, Porras y Carmona, 2006: 6).

También es importante contar con información objetiva para intervenir en los principales problemas y sus causas, otorgando firmeza al proceso de ejecución de las políticas y de implementación de planes de acción y soluciones acordes con estos, para facilitar la articulación de esfuerzos en aras de un mejor aprovechamiento de los recursos y de la obtención de resultados eficaces, como en el caso de la puesta de manifiesto y valoración de proyectos de inversión viables que redunden en la generación de actividad empresarial, renta y empleo (Carrillo, 2000: 69-71).

2 Estas etapas son: formación de la agenda de actuación de los poderes públicos, clasificación de los problemas o filtración de los problemas, definición de problemas, previsión (análisis de prospectiva), establecimiento de objetivos y prioridades, análisis de las alternativas, implantación de políticas, seguimiento y control, evaluación y revisión así como mantenimiento y reemplazo o terminación de políticas. 
Igualmente, la investigación social posibilita la evaluación de las acciones desarrolladas al facilitar el seguimiento y la monitorización de la ejecución operativa de las diferentes actuaciones, así como la medición de su impacto para conocer su eficiencia y verificar si los supuestos que se usaron, tanto para su formulación como para su implementación, fueron adecuados, incluyendo la identificación, análisis y explicitación de los cambios que se han producido como consecuencia de la aplicación de los proyectos (Angeleri, 1999: 15; ILPES, 2001: 15; Castro, 2002: 192; León, Porras y Carmona, 2006:3).

Sobre esta base, en el presente artículo pretendemos reflexionar acerca de las dificultades con las que se encuentra la administración local en la implementación del modelo para el desarrollo del territorio en el proceso de generación de informaciones procedentes de los programas implantados, que posibiliten contar con datos válidos para la mejora de los mismos. Consideraciones que a grandes rasgos pueden ser aplicables a las políticas públicas en ámbitos diferentes al del desarrollo local. En base a ello, las hipótesis del artículo serían las siguientes:

Las políticas de intervención en el ámbito del desarrollo local se implementan, en general, sin el análisis de la realidad específica y la problemática del entorno.

En los casos en los que el modelo de intervención pública en el desarrollo local mide sus efectos, lo hace sin contar con datos básicos o recurriendo a datos poco fiables, parciales o incompletos; en lugar de utilizar una cultura y una metodología para desarrollar buenos sistemas de observación e información sistematizada.

Al no establecer los mecanismos para la medición de su eficiencia (eficacia en su caso), el modelo de intervención pública en el desarrollo local no puede ofrecer los resultados más adecuados y las garantías suficientes para conseguir los objetivos propuestos.

\section{EL MODELO DE DESARROLLO LOCAL}

\section{Una breve reseña histórica}

La segunda mitad de la década de los ochenta supuso la aparición de un nuevo modelo para el desarrollo del territorio desde lo local; no obstante, la perspectiva macro daba muestras de su incapacidad operativa para reactivar la economía española tras la crisis vivida -inicialmente a nivel mundial- en la década de los setenta y posteriormente con su prolongación hasta finales de los ochenta. Ello dejaba paso a una actuación donde lo micro cobraba una importancia máxima.

El desarrollo de abajo a arriba (Alburquerque, 2002: 54) se convertía en la directriz básica para hacer frente a esta situación de crisis socioeconómica. Reactivar el

152 territorio suponía redefinir las actuaciones y las dinámicas desde lo pequeño. Lo rural 
-que había sido abandonado años atrás debido al avance del modelo de producción fordista- volvía a adquirir una dimensión fundamental. El desarrollo desde lo local es una concepción en la que la dinamización de los procesos económicos devuelve el protagonismo al territorio y a su población (Madoery, 2001: 125).

Estas estrategias de desarrollo endógeno (Vázquez Barquero, 1988, 2000a, 200ob; Pérez, 2000a: 117; Pérez y Carrillo, 2000; Rubio, 2000; Sanchis, 2000a: 158; Moltó y Hernández, 2002: 177) pretenden la activación de las potencialidades de los recursos y las dinámicas existentes en el ámbito local, y el fomento y la estimulación de la actividad productiva endógena de forma eficaz, coherente e integrada, complementándolos con las oportunidades que el entorno pueda generar para dar solución a los problemas concretos, en un enfoque territorial de abajo-arriba.

Asimismo, estas actuaciones constituyen una vía emergente para abordar de manera eficaz los procesos de desarrollo local (Esparcia, 2009: 272). Primero, diseñando respuestas imaginativas al problema del paro, entre las que se incluyen experiencias como las iniciativas locales de empleo que se han revelado eficaces en la creación de ocupación (Greffe, 1990; Vázquez Barquero, 1993: 201; Muiños y Cortegiano, 1998; Alós, 1999; Sánchez, 2000: 133; CCE, 2001; Banyuls et al., 2002). Posteriormente, elaborando estrategias de desarrollo local que ofrecen nuevos horizontes de promoción a sus comunidades (Salom, 2001: 13).

En este sentido, los recursos endógenos existentes en el territorio se convierten en los elementos centrales del nuevo modelo, por lo que su conocimiento es determinante para la consecución de sus objetivos.

Así lo recoge la norma reguladora de dichas iniciativas locales de empleo en su articulado. En concreto, desde la Orden Ministerial de 12 de abril de 1994, Reguladora de las Iniciativas Locales de Empleo y los Agentes de Desarrollo Local (Egea, 1999: 15; Calvo, 2010: 4-5), en las diferentes ordenaciones -como es el caso de la orden anual de la Conselleria de Economía, Hacienda y Empleo de la Generalitat Valenciana- se afirma que una de las funciones de los agentes de desarrollo local es la "prospección de recursos ociosos o infrautilizados, de proyectos empresariales e iniciativas locales de empleo" o afirmaciones muy similares. ${ }^{3}$

3 Con respecto a las funciones de esta figura profesional, si atendemos a la Orden de la Conselleria de Economía, Hacienda y Empleo, de 15 de julio de 1999, por la que se establecen las bases de concesión de subvenciones públicas para el fomento del desarrollo local e impulso de los proyectos y empresas calificados como I+E (Diario Oficial de la Generalitat Valenciana, 19 de octubre de 1999) o a la Orden de la Conselleria de Economía, Hacienda y Empleo, de 29 de diciembre de 2000 , por la que se que establece las bases reguladoras y el procedimiento general para la concesión de subvenciones del fomento del empleo dirigido a emprendedores (Diario Oficial de la Generalitat Valenciana, 25 de enero de 2001) y las siguientes, en éstas se indica que una de las funciones a desarrollar por los Agentes de Empleo y Desarrollo Local es la de "prospección de recursos ociosos o infrautilizados, de proyectos empresariales de promoción económica local e iniciativas innovadoras para la generación de empleo en el ámbito, identificando nuevas actividades económicas y posibles emprendedores". 
Analizar el territorio, para conocer las potencialidades del mismo y contar así con las informaciones suficientes en cantidad y calidad para la concreción de un plan estratégico de desarrollo territorial, son los objetivos que este nuevo modelo plantea como sus piedras angulares de funcionamiento.

Para el adelanto de las estrategias de desarrollo endógeno, por ejemplo, se postula como necesaria la detección y evaluación de los recursos autóctonos ociosos o infrautilizados, generadores de actividad económica y empleo, por medio de estrategias que intentan liderar los procesos de cambio estructural mediante el apoyo con servicios específicos, la gestión local y la utilización de agencias intermediarias de desarrollo que inciden en la mejora de la competitividad de los sistemas de pequeñas empresas locales y la transformación y flexibilización de un sistema económico diversificado, el crecimiento cualitativo de la productividad, la innovación y la calidad, el fomento de medidas activas del mercado de trabajo y de la capacidad emprendedora con el objetivo de crear puestos de trabajo cualificados y la mejora del nivel de vida, la ocupación y las rentas de la población (Vázquez Barquero, 1988, 1993: 201, 223, 1999: 52; CE, 2000; CCE, 2001; Boixader, 2007: 395; Esparcia, 2009: 271-273, 290; Calvo, 2010: 2-3). Y ello, aunque se trate de actuaciones complementarias, más que alternativas, a los enfoques más tradicionales y estén llenas de dificultades (Esparcia, 2009: 272-273, 290; Calvo, 2010: 10).

\section{La realidad del modelo}

Transcurridos veinticinco años desde sus inicios, la realidad no muestra una imagen muy distinta de la descrita en el apartado anterior. Presenta un modelo sin unos objetivos claros, que ha descentralizado a lo micro la ejecución burocrática y repetitiva de los programas, que no ha sabido dotarse de una estructura adecuada de funcionamiento que se ha ido configurando a sí misma con el transcurso de los años.

Un modelo que ha aplazado su faceta intervencionista y demorado su capacidad analítica, por lo que no ha contado con un sistema de información actualizado y continuo para la toma de decisiones. Es en sí un modelo en el que han tenido un papel fundamental las agencias de desarrollo local y, en especial, la figura de los agentes de empleo y desarrollo local -más conocidos como AEDL o simplemente ADL. Figuras que han marcado su evolución posterior (Sanchis, 2005).

De hecho, frente a estas carencias de información, desde la perspectiva del desarrollo local, lo que interesa saber, y planear, es cómo solucionar el problema de la generación de puestos de trabajo para ciudadanos concretos de determinado municipio, con sus capacidades y limitaciones, o conocer las consecuencias sobre el empleo y la renta que tiene el aumento de la producción de cierto sector económico, o las ventajas o inconvenientes de potenciar otro (Muiños y Cortegiano, 1998; Wilson, 2005: I). Y, también, conocer el número de empresas que se crean, cuántas son de 154 mujeres, cuántas empresas de servicios avanzados a empresas existen, cuántas aso- 
ciaciones hay, de qué tipología y qué necesidades manifiestan, etc. En otras palabras, estos datos son necesarios para conocer las necesidades de desempleados, emprendedores y asociaciones, para la selección de proyectos viables y para evaluar el diseño y puesta en marcha, por ejemplo, de una convocatoria de ayudas o el impacto de las actuaciones sobre la creación de empresas. Por ello, estas carencias de información dificultan establecer un perfil del desarrollo local.

A lo anterior hay que añadir una visión puesta en el corto plazo, buscando la justificación del gasto público realizado. El corto plazo se convierte en uno de los factores limitadores más importantes actualmente en el modelo de desarrollo local, ya que la definición de los programas se establece en dicha perspectiva. Este aspecto imposibilita el manejo adecuado de las actuaciones por parte de las agencias locales de empleo, e impide a su vez la obtención de los niveles de eficiencia deseados (Calvo y Lerma, 2008: 35-36; 2009: 19).

También la propia concepción inicial del técnico AEDL, como dinamizador y generador de empleo, se establece en términos economicistas y poco sociales (Calvo y Lerma, 2008: 34). Se sigue manteniendo la concepción originaria del técnico en los años ochenta, donde se requería una formación preferiblemente en Derecho o Economía que encauzaba la futura orientación de la actividad del agente. Junto a ello, la exigencia institucional de consecución de resultados cuantitativos en el corto plazo, supone un freno a una verdadera actuación en el terreno social.

La ausencia de directrices claras en el desarrollo de la actividad del AEDL, que queda en manos de la orientación personal del técnico es otra realidad del modelo. El agente de empleo y desarrollo local parte sin una definición clara y concisa de su actuación profesional, lo que supone una heterogeneidad muy elevada en lo referente a la orientación seguida en la actividad del técnico. Queda por tanto a su libre disposición, o en su caso del político responsable, la concreción del alcance y de los contenidos prácticos de su trabajo (Calvo y Lerma, 2008: 36).

La organización misma del trabajo de una agencia local de empleo imposibilita y limita la planificación adecuada de la actividad. Una escasa atención a la búsqueda de indicadores sociales de referencia muestra las debilidades internas de un modelo como el implantado. Los técnicos actúan en muchas ocasiones basándose en su intuición, voluntad o predisposición, sin contar con una base de información técnico-científica que asegure el éxito de la intervención (Calvo y Lerma, 2008: 36).

Los objetivos de los AEDL se establecen en términos básicamente cuantitativos y poco cualitativos. Dada la concepción de la actividad de estos profesionales, ellos responden al cumplimiento de unos objetivos definidos cuantitativamente -en número de programas de empleo desarrollados, en volumen de recursos económicos conseguidos para la localidad, en cantidad de empresas creadas o de parados insertados en su caso- sin plantear una cuestión cualitativa de fondo: la verdadera utilidad de las actuaciones realizadas, definida ésta en términos de, por ejemplo, la duración 
o vigencia de los contratos de trabajo de los parados insertados, la evolución y necesidades actuales de los proyectos empresariales puestos en funcionamiento, la necesidad de programas de empleo adecuados a la realidad municipal, etc. (Calvo y Lerma, 2009: 19-20).

En definitiva, la mejora social de los programas de empleo se presupone más que se demuestra, puesto que la inexistencia de unos indicadores claros impide afirmar rotundamente su existencia, aunque la presumimos como existente. Por ello, la escasa atención a la búsqueda de indicadores sociales de referencia que permitan conocer en mayor grado el contexto social en el que van a desarrollar su actividad, definen la débil situación de partida de las actuaciones realizadas. Los programas de empleo son definidos en niveles muy lejanos a la realidad donde van a ser aplicados, lo que supone la aparición de una serie de desviaciones y de pérdida muy amplia de esfuerzos y recursos.

\section{LOS PROBLEMAS DE LAS ADMINISTRACIÓN PÚBLICA EN LA GENERACIÓN DE INFORMACIONES SOBRE SUS ACTUACIONES EN EL ÁMBITO DEL DESARROLLO LOCAL}

Sobre la base de las reflexiones planteadas hasta el momento por diferentes estudiosos de la materia, y de los resultados de un estudio empírico en curso realizado en la Comunitat Valenciana, que tiene por objetivo principal analizar los efectos que las políticas de empleo y desarrollo local han generado sobre el territorio (Calvo y Lerma, 2008: 18-21; Calvo y Lerma, 2009: 34-38; Calvo, 2010: 10-16; Calvo y Aguado, 2011: 19-24), puede afirmarse que en la actualidad existen importantes carencias públicas en generación de información, sobre todo en el modelo de desarrollo local, que dificultan su conversión en conocimiento útil para la acción, como las que describimos a continuación en relación al modelo de desarrollo local indicado:

a) Dicho modelo se caracteriza por la inexistencia de unos indicadores de gestión válidos y validados, y su no concreción en unos mecanismos e instrumentos adecuados para el aprovechamiento correcto y útil de la información generada en sus actuaciones.

b) El modelo público de desarrollo local adolece del fomento de un proceso retroalimentado que facilite la mejora del mismo. La falta de costumbre generalizada de la administración pública española y en algunos casos su escasa implicación en la materia son elementos que adquieren una gran importancia.

c) El factor anterior se convierte en un miedo a los posibles resultados que genere la medición, ya que es posible que el proceso de revisión del sistema nos indique una cierta ineficiencia pública en la gestión de los recursos invertidos sobre el territorio o, en cualquier caso, nos daría una visión real de cuál es la capacidad de las políticas públicas de intervenir sobre el territorio. 
d) Los procesos administrativos han derivado en una burocratización de los procesos de actuación pública, lo que a su vez ha conllevado un rasgo de repetitividad muy elevado de los programas desarrollados.

e) La inexistencia de un liderazgo institucional definido y establecido en la materia, cuestiona su viabilidad y plantea la eterna cuestión: ¿quién toma la iniciativa?, ¿se trata de un sistema que se debe diseñar de arriba a abajo? o por el contrario, ¿se debe retroalimentar automáticamente de abajo a arriba?

f) Por otra parte, ¿en qué grado está preparada nuestra administración pública actual para la implementación de procesos de evaluación de sus actuaciones? La capacidad de coordinación entre los distintos niveles implicados, la existencia de canales y herramientas, el grado de preparación y motivación del personal, y la concienciación respecto de su utilidad -presente y sobre todo futura-, son elementos de debate que habría que plantearse.

g) Muy relacionado con ello se encuentra un factor como la escasa profesionalización del proceso aplicado para la gestión del bien público, que pone mayor énfasis en el desarrollo de la ejecución que en la articulación de un sistema de control, seguimiento y evaluación del mismo (que servirá como elemento nuclear de la planificación posterior).

h) Es, por tanto, un modelo que formaliza lo informal como metodología de actuación, que elude los procedimientos formales para la consecución de informaciones, pero que valida en el día a día las informaciones obtenidas informalmente.

i) Por último, otro factor importante es la inexistencia de una planificación estratégica adecuada, que dificulta la aplicación de una visión integral de conjunto para la obtención del bien común en el ámbito del desarrollo local.

Debido a estos rasgos del modelo de desarrollo del territorio, a menudo el análisis realizado por los gestores de la administración resulta parcial y sesgado, más o menos mediado por valores, emociones, juicios y prejuicios, por afinidades culturales, por los usos que cada cual hace del territorio, por las relaciones entre los diferentes grupos e individuos, por el contacto desigual con los demandantes de diferentes obras y servicios, tal como indica Morales (1997), en la descripción de un sistema de indicadores regionales, y por otros condicionamientos, no solamente por un preciso cálculo de interés propio. Cada uno mira el mundo con ojos diferentes, funcionando como "metáforas" o "faros" que centran su atención en lo que consideran necesario analizar o dónde desean actuar y dejan en la sombra otros rasgos o fenómenos que podrían ser relevantes. Actúan de forma semejante a los seis ciegos hindúes de la parábola clásica, donde cada cual colocaba su mano en una parte diferente del elefante y lo describía como un animal distinto (Gusfield, 1994: 93-94, 113, Laraña, 1999: 17-18). 
Todo ello implica la inclinación hacia las decisiones intuitivas o precipitadas, la ausencia de una cultura y una metodología para desarrollar buenos sistemas de observación e información sistematizada y la pérdida de datos básicos, importantes para definir las políticas municipales más adecuadas para cada objetivo. Así lo afirman diferentes estudiosos del desarrollo local en sus textos (Angeleri, 1999: 15; Sanchis, 2000a: 168, 186 y ss.; 200ob: 287) y en sus estudios empíricos (Sanchis et al., 2001: 23). Ello se debe, por ejemplo, a la incompleta información sobre las tendencias de los mercados de trabajo o de los sectores económicos. De hecho, esto tiene como consecuencia que la toma de decisiones no siempre responda a criterios de eficiencia, equidad, pertinencia, coherencia, consistencia, sostenibilidad, responsabilidad, transparencia o eficacia, y que los criterios con los cuales se elaboran, evalúan y ejecutan los programas ofrecidos no se ajusten a las prioridades o necesidades básicas de la población existente en el territorio (ILPES, 2001: 9; Calvo, 2010: 11).

Además del déficit de información y de datos estadísticos, cuando éstos existen son poco fiables, parciales o incompletos, incluso en aspectos muy básicos, algo que sólo muy reciente y parcialmente empieza a solucionarse. Estas afirmaciones son compartidas, en mayor o menor grado, por Castro (2002: 198) en su tesis doctoral sobre la aplicación para Andalucía de diferentes indicadores de desarrollo sostenible urbano, por Batet et al. (2003: 7) en su propuesta de indicadores básicos de gestión de servicios públicos locales o por Sevilla (2006:2) en su ponencia sobre la experiencia en la Seguridad Social para la utilización de los registros administrativos con fines estadísticos. Pero lo destacable es que todo ello puede inducir a errores sobre las posibilidades del territorio si las conclusiones que arrojan las cifras no son debidamente relacionadas con otras variables (Wilson, 2005: I; García Rodríguez, 2009: 11).

A ello hay que añadir que, aunque la información exista, en la mayoría de los casos ésta es aplicable a escalas superiores en términos agregados que responden a los objetivos de representación estadística macrorreferencial, con relación al Estado o, en el mejor de los casos, a las comunidades autónomas o las provincias -producción, valor añadido, empleo, niveles de ingreso, estructura de gastos, fuentes laborales, etc.-, incluyendo diferencias en los métodos analíticos y en las formas de reaccionar a los problemas de cada territorio que no coinciden con la escala municipal de toma de decisiones (Castro, 2002: 2, 198; Wilson, 2005: I). Por ello, en el ámbito local, los instrumentos para cuantificar el desarrollo se difuminan, haciendo que los datos y los análisis de tipo social o microeconómico sean escasos, inadecuados y muy heterogéneos, más allá de las variables demográficas derivadas de censos y padrones (Castro, 2002: 2).

En otras ocasiones, como indica Aguado (2010: 5), la información existe pero no se le brinda a quien la requiere, no se puede acceder a algunas fuentes externas de información -por ejemplo, Seguridad Social o Hacienda- o su coste es muy elevado 158 -por ejemplo, Registro mercantil-, por lo que es como si no existiera. 
A lo anterior hay que añadir las dificultades que suponen la ambigüedad en cuanto al significado de los indicadores o la disociación entre estos y el fenómeno a medir, la heterogeneidad de las fuentes estadísticas, la dificultad práctica de incluir indicadores subjetivos o de percepción, la escasa cultura e implicación en la evaluación, control o seguimiento de los programas desarrollados o el hecho común de que se carezca de una visión precisa sobre el tipo de información que se requiere, la forma en que ésta será procesada y, finalmente, de su utilidad dentro de las tareas de gobierno (Morales, 1997: 1; Castro, 2002: 198; Batet et al., 2003: 8; Calvo, 2010: 12). Todo ello, sin olvidar el problema de que las diferentes agrupaciones de información no son equiparables en términos de variables y metodologías utilizadas, haciendo imposible la comparación temporal y espacial (Castro, 2002: 3, 198).

Las afirmaciones precedentes sobre la falta de mediciones por parte de la administración pública en el ámbito del desarrollo local, se corroboran desde diferentes puntos de vista por los estudiosos de la materia:

Desde una perspectiva teórica, en sus tesis sobre los programas, servicios y funciones de las agencias de desarrollo local, distintos autores destacan la necesidad de proyectos para detectar y evaluar el potencial de los recursos endógenos del territorio mediante la realización de estudios socioeconómicos y similares (Muiños y Cortegiano, 1998; Carrillo, 2000: 69-71; Pérez y E. Carrillo, 2000: 55; Sánchez, 2000: 275; Sanchis, 2000a: 183; Sanchis et al., 2001: 21).

Llegamos a conclusiones similares si consideramos, desde un punto de partida formal, las características de los agentes de inserción sociolaboral, de empleo o de desarrollo local (Sanchis, 2000a: 220), sus rasgos profesionales (Sanchis et al., 2001: 36; Esparcia, 2009: 274) o sus funciones (Sánchez, 2000: 273). En estos textos se destaca, a grandes rasgos, que estos profesionales son -o deben ser- investigadores y conocedores de las problemáticas, las necesidades, los recursos y las demandas económicas y sociales de la localidad en la cual actúan, así como transmisores de esta información a los diferentes agentes socioeconómicos para promocionar actividad económica que genere empleo (APPEDL, 1997; Sanchis, 2000a: 96; Sanchis et al., 2001: 36; Esparcia, 2009: 274, 276-277, 278-281; Calvo, 2010: 6).

Desde el punto de vista empírico, de acuerdo con lo indicado, los encuestados de la investigación de Sanchis et al. (2001), pertenecientes a estas figuras profesionales, dan importancia a la realización de estos estudios en su trabajo (23,5\%) que es superior a la asignada a la realización de trámites (17,7\%) y a la gestión interna de oficina (5,9\%); y similar a la de gestión de recursos $(23,5 \%)$, orientación sociolaboral (23,5\%) y difusión de información (23,6\%). En el mismo sentido, puede destacarse la demanda de formación en metodología y técnicas de investigación social por parte de estos profesionales (FORCEM, 2000: 77) y la carencia de agentes de desarrollo local con formación específica en este tema (APPEDL, 1997). 


\section{LA EXPERIENCIA DEL MUNICIPIO DE CATARROJA COMO REFERENTE}

Frente a este contexto, una excepción que confirma la regla es el caso del sistema de información permanente de la realidad socioeconómica, desarrollado por el Servicio de Desarrollo Local y Participación Ciudadana del Ayuntamiento de Catarroja, un municipio de 28.223 habitantes del área metropolitana de Valencia.

\section{El sistema de información socioeconómica}

Las agencias de desarrollo local, como parte de las entidades locales, están en condiciones de convertirse en un observatorio de la realidad socioeconómica del área, mediante la recogida, sistematización, el análisis y la estructuración de la información sobre la realidad en la cual se actúa como es el caso que se presenta (SDLPC, 2009), por lo tanto, en una de las principales fuentes de información para los agentes institucionales, económicos y los colectivos sociales del municipio (Morales, 1997: 1; Castro, 2000: 29; Pérez, 2000a: 119-120; 2000b: 19) ante las nuevas problemáticas y demandas sociales.

Se trata de un servicio en gran parte intangible (Ballesteros, 1999: 48; Sanchis, 2000c: 850, 874; Sanchis, 2000a: 189; Hernando, 1999: 12), a la vez que suficientemente flexible para adaptarse a las necesidades y los diferentes tipos, niveles de desarrollo y tamaño de municipios, que puede fortalecer permanentemente la creación, el tratamiento y los métodos de obtención de los datos y la oferta de un recurso de naturaleza simbólica, como la información, de manera elaborada y estructurada.

En concreto, este servicio del Ayuntamiento de Catarroja tiene como finalidad el fomento de la creación de actividad económica y empleo en la población mediante el asesoramiento a la creación de empresas, la orientación en la búsqueda de empleo, el apoyo al sector empresarial, la promoción del comercio local y del uso de las nuevas tecnologías. Para ello articula un sistema de información, herramienta que permite la configuración de una estructura integrada y racional de indicadores que habilita para obtener conocimiento sintético de los fenómenos a partir de datos primarios producidos por la propia entidad, como de datos secundarios proporcionados por organismos dedicados a tal labor, tanto en general como sectoriales, como se describe seguidamente en el plan de trabajo y la metodología del programa. En nuestro caso, sobre la evolución y la dinámica de los principales aspectos del municipio y de su entorno, obedeciendo a una construcción teórica concreta y a un análisis previo a su elaboración (Castro, 2002: 196-197; León, Porras y Carmona, 2006: 6).

Concretamente, los objetivos del observatorio socioeconómico son: 1) la construcción de un sistema de indicadores, 2) que permita recoger información socioeconómica del municipio y de su entorno, sistematizarla y estructurarla, diseñar herramientas para facilitar la interpretación de los datos, analizarlos y divulgar datos 160 objetivos y un conocimiento detallado y permanente de la realidad sobre la que se 
actúa a los clientes tanto internos como externos, y 3) que se enmarque dentro del mapa de procesos del Ayuntamiento.

\section{Plan de trabajo y metodología}

\section{- Antecedentes y marco}

El antecedente más inmediato del observatorio socioeconómico es el Estudio Socioeconómico de Catarroja 2000-2001. Tras la realización del mismo, la meta del SDLPC es que los estudios para descubrir huecos de mercado que se están elaborando sobre actividades económicas, población desempleada, etc., puedan convertirse en algo que se haga de manera sistemática y se puedan tener datos continuamente actualizados para evitar desfases, mediante la creación de un observatorio socioeconómico permanente de Catarroja.

En este estudio socioeconómico se llevó a cabo un primer avance de la construcción de un sistema de indicadores, el cual incluyó la recopilación y el análisis de datos de diferentes fuentes externas -Instituto Nacional de Estadística (INE), Instituto Valenciano de Estadística (IVE), Servicio Valenciano de Empleo y Formación (SERVEF), Agencia de Desarrollo Comarcal, etc.- e internas -Impuesto de Actividades Económicas (IAE), Padrón Municipal de Habitantes, etc.- y una primera sistematización y compilación de indicadores.

Por otra parte, el observatorio socioeconómico se enmarca en un proyecto más amplio, de manera complementaria al Plan Estratégico de Catarroja que elaborará un sistema de indicadores sobre el desarrollo del municipio y que incluye unas 400 encuestas a los ciudadanos del municipio y un diagnóstico mediante la técnica del DAFO.

\section{- Metodología}

Atendiendo a la metodología, los pasos seguidos para el desarrollo e implantación del sistema de información socioeconómica han sido los siguientes:

En primer lugar, se ha realizado el análisis de las fuentes documentales municipales, como la matrícula del IAE, el padrón municipal, las licencias de obra mayor, etc. En este estudio de las fuentes de datos se ha considerado la fecha y la frecuencia de actualización de los mismos, el plan de explotación a desarrollar y la "calidad" de los datos que describimos seguidamente.

En segundo lugar, se ha buscado información de otros observatorios y de otras fuentes, como es el caso de las estadísticas del SERVEF, los datos del INE, del IVE o de las Fundaciones de La Caixa y Caja España, entre las más destacadas. Asimismo, siguiendo la técnica del benchmarking, se han tenido en cuenta otros observatorios socioeconómicos, tanto específicos - del mercado de trabajo- como generales, lleva- 
dos a cabo tanto por instituciones como por el denominado Tercer Sector, así como, también, otros estudios socioeconómicos existentes. ${ }^{4}$

En tercer lugar, se ha diseñado el sistema de información y el análisis de los indicadores. En nuestro caso, se ha pretendido que los indicadores resultantes cumplan los siguientes rasgos o criterios de calidad: sencillos, simples, claros y comprensibles; fácilmente obtenibles; con validez científica; fiabilidad; medibles; representativos (han de permitir la especificación empírica de conceptos que no pueden ser completamente medidos de forma operativa); relevantes (han de proporcionar información destacada); comparables; flexibles (perfeccionamiento progresivo); modulables (fácil incorporación de nuevas relaciones y jerarquías); con el mayor nivel de desagregación territorial; orientados a colectivos; informatizados y automatizados; estables; eficientes (económicamente efectivos con relación al coste de obtención de datos y del uso de la información que aportan) y predictivos (Otero i Serra, 1998: 3; Arriagada, 2002: 58; Castro, 2002: 194-196; Batet et al., 2003: 6; León, Porras y Carmona, 2006: 4).

En cuarto lugar, se procede al desarrollo de un plan de análisis de dichos indicadores considerando la fuente de datos (padrón municipal, servicios municipales de gestión tributaria, de urbanismo, de educación u otros, INE, IVE, SERVEF), su dimensión (demografía, actividad económica, mercado de trabajo, formación, etc.), sus componentes (población, migración, niveles de formación, datos sobre sectores económicos, paro registrado, etc.), los indicadores (género, edad, barrio, país, nivel de formación, altas y bajas de actividades, paro por edad, sexo, sector, etc.), su periodicidad (mensual, semestral, anual, etc.).

Por último, tras el trabajo previo, se lleva a cabo la creación de índices y/o ratios (demográficos, de actividad económica, de desempleo, etc.).

\section{Estudios realizados en el entorno local}

La necesidad de contar con información suficiente que garantice el éxito de las posteriores actuaciones emprendidas, y el compromiso de la Corporación local con el desarrollo del territorio, ha llevado a que desde el Servicio de Desarrollo Local y Participación Ciudadana (SDLPC) se hayan elaborado investigaciones en ámbitos como la planificación estratégica y participación ciudadana; evaluación de los servicios mu-

4 Entre los observatorios cabe destacar los siguientes: Observatorio socioeconómico de Segovia. <http://www.cajasegovia.es/observatorio/identifi/identifi.htm>; Observatorio socioeconómico de Cádiz <http://www.uca.es/grup-invest/dgt/paginas/observatorio.html>; Red de Observatorios del Mercado de Trabajo Local de la provincia de Barcelona <http://www.diba.es/promocio_economica/bones_practiques/ actuaes/ fitxa23.htm> que incluye, por ejemplo, L'Observatori Econòmic i Social de Terrassa. Entre los estudios, por ejemplo, los de Rivera (2003), Ramírez (2003) y Servicio Andaluz de Empleo (2003) realizados, todos ellos por las Unidades Territoriales de Empleo, Desarrollo Local y Tecnológico de Andalucía; asimismo el del Consejo Económico y Social de Jerez (2004), disponible en <http://www.jerezserviciosempresariales.com/informese/ informes/boletin2004.pdf>; o el de la Mancomunidad Intermunicipal de L'Horta Sud de Valencia (2003). 
nicipales y calidad; estudios e informes socioeconómicos y análisis del impacto de las tecnologías de la Información y la Comunicación sobre la realidad local.

Por ello, además de los estudios socioeconómicos propiamente dichos, basados en las fuentes secundarias mencionadas, dentro del observatorio se han realizado diferentes investigaciones monográficas -tanto cuantitativas como cualitativas- sobre distintos ámbitos sociales y económicos del municipio. Han sido estudios de carácter primario mediante la aplicación de técnicas de investigación social: encuestas con cuestionario precodificado, entrevistas en profundidad, grupos de discusión, observación participante, etc. Un nivel interno de investigación que se ha complementado con la demanda por parte de la Corporación de diferentes investigaciones a consultoras externas o universidades (de Valencia, Politécnica de Valencia) sobre la realidad local. Tal es el caso de la Encuesta bienal de Calidad de Vida, el Plan de Acción Comercial, la Encuesta de Satisfacción de los servicios públicos o el Plan Estratégico “Catarroja 2010" (ver Cuadro 1).

\section{CUADRO 1}

Estudios realizados por el SDLPC - Catarroja (2002-2010)

\begin{tabular}{|c|c|}
\hline $\begin{array}{l}\text { Planificación estratégica y } \\
\text { participación ciudadana }\end{array}$ & $\begin{array}{l}\text { - Plan Estratégico de Catarroja } 2010 \\
\text { - Consultas ciudadanas } \\
\text { - Plan de Acción Comercial } \\
\text { - Otros }\end{array}$ \\
\hline $\begin{array}{l}\text { Evaluación de los servicios } \\
\text { municipales y calidad }\end{array}$ & $\begin{array}{l}\text { - Plan de mejora continua y Plan de calidad total (EFQM). } \\
\text { Creación y mantenimiento del Sistema de Indicadores de } \\
\text { calidad del SDLPC } \\
\text { - Encuesta Calidad de vida y satisfacción con los servicios } \\
\text { - Otras encuestas de Satisfacción de los servicios públicos } \\
\text { - Evaluación de los servicios municipales. Oficina de Aten- } \\
\text { ción al Ciudadano, Servicios a las personas. } \\
\text { - Otros }\end{array}$ \\
\hline Estudios socioeconómicos & $\begin{array}{l}\text { - Estudios socioeconómicos bienales } \\
\text { - Encuesta anual a los usuarios del servicio de creación de } \\
\text { empresas } \\
\text { - Estudios del tejido empresarial del polígono } \\
\text { - Guía comercial y de servicios del núcleo urbano } \\
\text { - Otros }\end{array}$ \\
\hline Otros estudios sociales & $\begin{array}{l}\text { - Estudio sobre jóvenes y comercio local } \\
\text { - La Catarroja dels majors: estudio sobre la población ma- } \\
\quad \text { yor de la localidad }\end{array}$ \\
\hline $\begin{array}{l}\text { Tecnologías de la Información } \\
\text { y la Comunicación }\end{array}$ & $\begin{array}{l}\text { - Asociacionismo y tecnologías de la información } \\
\text { - Mujeres y tecnologías de la información }\end{array}$ \\
\hline Informes socioeconómicos & $\begin{array}{l}\text { - Implantación de proyectos empresariales en la localidad } \\
\text { y proyectos I+E } \\
\text { - Relacionados con actividades municipales: proyectos de } \\
\text { la UE, educativos, otros } \\
\text { - Otros informes }\end{array}$ \\
\hline
\end{tabular}

Fuente: SDLPC-Catarroja 


\section{Difusión del observatorio socioeconómico}

La difusión del observatorio se lleva a cabo de la siguiente forma, que describimos de forma esquemática respondiendo a diversas preguntas clave:

Qué: el boletín periódico, tanto electrónico (e-mail) y página web <http://www. catarroja.es> como en formato papel (según el estudio concreto).

A quién: a los/as trabajadores/as de la administración local, a los/as emprendedores/as, empresarios/as y representantes de los/as agentes económicos (asociación de comerciantes, de empresarios del polígono, sindicatos, etc.) y a la población en general.

Cómo: mediante las nuevas tecnologías de la aldea global electrónica (página Web municipal y correo electrónico) y las de la galaxia Gutenberg (papel).

Cuándo: según la periodicidad acordada (según la periodicidad de los estudios, generalmente bienal).

Dónde: sobre todo en Catarroja y en la Mancomunitat de l'Horta Sud.

\section{A MODO DE CONCLUSIÓN: LA ADMINISTRACIÓN LOCAL Y EL ANÁLISIS DE LAS POLÍTICAS PÚBLICAS DE DESARROLLO LOCAL}

Tras la exposición previa, cabe indicar en relación con las hipótesis de partida que las tres se corroboran.

En primer lugar, hemos observado, tanto de forma teórica como empírica, cómo las políticas de intervención en el ámbito del desarrollo local se implementan sin un correcto análisis de la realidad específica y la problemática del entorno antes de actuar o sin conocer sus resultados (efectos).

Asimismo, en el modelo de intervención socioeconómica en el territorio, la administración pública no cuenta con una cultura y metodología para desarrollar buenos sistemas de observación e información sistematizada, sino que se deja en manos de la voluntad local que se midan sus efectos con ausencia de datos básicos o siendo estos poco fiables, parciales o incompletos.

Por último, se ha puesto en evidencia cómo el modelo de intervención descrito no puede ofrecer los resultados más adecuados y las garantías suficientes para conseguir los objetivos propuestos cuando no se establecen los mecanismos para la medición de su eficiencia (eficacia en su caso).

No obstante, también hemos visto cómo se atisban espacios de luz en este oscu164 ro panorama, pues la experiencia descrita muestra cómo pueden y deben crearse 
nuevos contextos de actuación para la investigación social en el ámbito del desarrollo local, una oportunidad para estas políticas dentro de estas transformaciones que experimenta la administración local.

En concreto, los aspectos novedosos del observatorio y la oportunidad del mismo, que mostramos a continuación, se convierten en una muestra del cambio de la gestión de la organización municipal (Arriagada, 2002: 14), al menos en ámbitos como el del desarrollo local. En concreto, desde una visión prospectiva y estratégica (Muiños y Cortegiano, 1998) y una postura proactiva (Cárdenas, 2002: 68), se ha coadyuvado en la modificación de las políticas de la administración local hacia la receptividad administrativa (MAP, 2000). Esto es, en el municipio de referencia, la implementación del sistema de información se ha dado de forma simultánea a la introducción de un programa de calidad total de la administración siguiendo el modelo EFQM (European Foundation for Quality Management), la certificación parcial del SDLPC en la norma ISO 9001:2000 y la realización del Plan Estratégico de la ciudad y del de la administración municipal.

De hecho, en este marco, la incorporación del sistema de información socioeconómica al servicio de desarrollo local es un paso "lógico", una oportunidad para las políticas de desarrollo local dentro de las transformaciones que ha experimentado la administración municipal como una herramienta de análisis y de programación de una administración proactiva en su preocupación por mejorar la gestión en los servicios públicos, mediante un proceso de adaptación de éstos a las necesidades de la ciudadanía. Esto es, una administración acompasada con las transformaciones económicas y sociales que se van produciendo, cuya meta es la atención de las nuevas demandas sociales descubiertas como, por ejemplo, la detección y evaluación de los recursos endógenos ociosos o infrautilizados de la localidad, generadores de actividad económica y empleo. Todo ello, haciendo especial énfasis en los nuevos estilos de gestión pública y en la necesidad de aumentar los grados de economía, eficacia, eficiencia, coordinación, transparencia y responsabilidad (PNUD, 2000: 89; Arriagada, 2002: 13-14; Batet et al., 2003: 5, 17; León, Porras y Carmona, 2006: 3).

En este sentido, la experiencia descrita muestra cómo la administración pública contemporánea debe basar su servicio público en criterios de:

a) Novedad e innovación, nuevas funciones de la administración, desarrollo local endógeno, ventajas de ofrecer un servicio como este, etc. Factores que ponen de manifiesto que las agencias de desarrollo local son el ámbito adecuado para llevar a cabo esta actuación. Ello las faculta para convertirse en una de las principales fuentes de información de la población y, en especial, de empresarios y emprendedores.

b) Base de datos obtenidos científicamente, lo que no puede medirse -cuantificarse- no existe (es el ejemplo del observatorio socioeconómico planteado que nos permite hablar con objetividad). 
c) Conocimiento relevante para la acción de la administración local mediante el análisis de los principales vectores que concurren en los procesos de desarrollo socioeconómico. Se trata de una forma de investigación para la planificación de las respuestas de la Corporación a las demandas/necesidades sociales.

d) La importancia del trabajo realizado en cuanto a que los programas aplicados se ajustan en mayor medida a la realidad del territorio en el que son desarrollados, con lo que además se consigue un mayor nivel de intervención social, de mejora y de progreso de la comunidad.

e) Anticipación a los problemas, lo que permite a medio y largo plazo ser más flexibles y adaptarse a las tendencias sociales y económicas, y a las exigencias que establece el cambiante entorno actual.

f) Calidad en el servicio, la mejora de la información, actualizada de manera periódica, la adaptación del servicio a los usuarios y la determinación de los diferentes procesos de actuación a seguir en los distintos programas.

g) Mejora de la satisfacción de los usuarios de los servicios públicos ofrecidos tanto usuarios entendidos directamente como ciudadanos o indirectamente como del resto de departamentos de la propia Corporación local- y, por lo tanto, de un mayor grado de la calidad percibida por ellos. Se aumenta la retroalimentación interna del sistema de trabajo -que ayuda a todo lo anterior, sobre todo al mejor cumplimiento de los objetivos-, mejora la imagen del servicio público -que en muchas ocasiones al ser de carácter gratuito desemboca en una imagen degradada del mismo- y, además, todo ello supone una mayor justificación de los recursos invertidos en el proceso de implementación de las políticas activas de empleo.

h) Consecución de una mayor eficiencia de la actividad desarrollada en las agencias de desarrollo local, manifestada principalmente en el grado de cumplimiento de los objetivos propuestos, lo que redunda en el hecho de disponer de datos y de información suficiente para la elaboración de las memorias e informes anuales -de carácter obligatorio para dichas entidades- y que sirven, a su vez, como una proyección exterior de la imagen de la actividad desarrollada.

i) Mejora de la capacidad para justificar la labor ante los órganos oficiales -dado que se cuenta con mayor cantidad y calidad de informaciones sobre las acciones y programas de empleo desarrollados. Esta mejor justificación del trabajo desarrollado supone, a su vez, una oportunidad clara y manifiesta de ampliar las líneas de actuación en materia de empleo, a través de la renovación de los programas o de su ampliación -en número e incluso en dotación 166 económica. 
j) Detección y evaluación de los recursos endógenos y nuevos yacimientos de empleo existentes en el territorio, lo que, a su vez, permite el contacto con los agentes económicos y sociales. La difusión del observatorio socioeconómico es un mecanismo de comunicación con los actores económicos y sociales.

k) Favorecer a las empresas y a los emprendedores, sirviendo de soporte a las empresas y a las actividades emprendedoras, mediante la aportación de datos y de estudios de mercado.

I) Transversalidad de la administración, permitiendo la actuación horizontal del conjunto de las áreas de la misma, pues facilita el diseño de procesos de creación de protocolos de actuación. Esto es, permite crear procedimientos por los cuales la información fluye de unos departamentos a otros de manera definida en dicho proceso, según la frecuencia de actualización de la información, su disponibilidad, etc.

\section{REFERENCIAS BIBLIOGRÁFICAS Y DOCUMENTALES}

Aguado, J.A. (2010), “La investigación social y los sociólogos como profesionales en el ámbito del desarrollo local: el caso de un municipio del área metropolitana de Valencia", en X Congreso Español de Sociología. Pamplona: Federación Española de Sociología.

Alburquerque, F. (2002), Desarrollo económico territorial. Guía para agentes. Sevilla: Instituto de Desarrollo Regional y Fundación Universitaria.

Alós, R. (1999), “Las políticas de desarrollo local en Cataluña”, Papers, 58: 75-96.

Angeleri, G. (1999), "El desarrollo económico local como herramienta para el desarroIlo social”, Observatorio Social, 3: 15-16. En línea: <http://www.redelaldia.org/ IMG/pdf/El_desarrollo_economico_local_como_herramienta_para_el_desarrollo.pdf> - <http://www.uniendopueblo.com.ar/documentos/ eldesarrolloeconomicolocal.zip> (consulta: 25 octubre 2011).

APPEDL (1997), Mancances de les Agències de Desenvolupament Local i els seus agents. Valencia: Associació Professional per a la Promoció Econòmica i el Desenvolupament Local. Inédito.

Ariño, A. (1997), Sociología de la cultura. La constitución simbólica de la sociedad. Barcelona: Ariel.

Arriagada, R. (2002), Diseño de un sistema de medición de desempeño para evaluar la gestión municipal: una propuesta metodológica. Santiago de Chile: Instituto Latinoamericano y del Caribe de Planificación Económica y Social - ILPES y CEPAL.

Asensi-Artiga, V. y Parra-Pujante, A. (2002), "El método científico y la nueva filosofía de la ciencia", Anales de Documentación, 5: 9-19.

Asociación Profesional de Formación y Empleo (2000), Desarrollo Local y Empleo en Tiempos de Globalización. Granada: Diputación Provincial de Granada y CajaGranada. 
Ballesteros, M. (1999), “El futuro del desarrollo local”, en Comunicaciones del curso Estrategias para la promoción económica y el empleo. Valencia: Universidad Internacional Menéndez Pelayo.

Banyuls, J., Cano, E., Picher J.V. y Sánchez, A. (2002), "Condicions de treball en els nous jaciments d'ocupació", Quaderns de Ciències Socials, 6: 1-54.

Batet, M., Pastor, J.R., Carreras, J.M. y Mora, A. (2003), Propuesta de indicadores básicos de gestión de servicios públicos locales, Document Pi i Sunyer, 23. Barcelona: Fundació Carles Pi i Sunyer d'Estudis Autonòmics i Locals.

Becerra, F., El Huajibi, H., Morilla, F., Muñoz, J.A., Nieto, E. y Villalba, F. (2004), Jerez: economía y sociedad 2004. Málaga: Consejo Económico y Social de Jerez de la Frontera. En línea <http://www.jerezserviciosempresariales.com/informese/informes/boletin2004.pdf> (consulta: 25 octubre 2011).

Boixader, J. (2007), “Estrategias para el desarrollo económico local. Aproximación a la provincia de Barcelona desde el modelo SLOT", Revista OIDLES, 2(1): 394-421. En línea: <http://www.eumed.net/rev/oidles/02/Boixader.zip> (consulta: 25 octubre 2011).

Calvo, R. (2010), “La escasa implicación de la Administración como freno al desarroIlo económico del territorio: el estudio del caso valenciano", en XII Jornadas de Economía Crítica. Zaragoza: Economía Crítica. En línea: <http://www.ucm.es/ info/ec/jec12/archivos/A2ECONOMIA\%20REGIONAL\%20Y\%20TERRITORIO/ ORAL/CALVO\%2OPALOMARES/CALVO\%20PALOMARES.pdf> (consulta: 25 octubre 2011).

Calvo, R. y Lerma, I. (2009), “Desarrollo local: ¿clientelismo subvencionado? Conclusiones críticas al modelo actual”, Acciones e Investigaciones Sociales, 25: 5-22.

Calvo, R. y Lerma, I. (2008), "Políticas de empleo a nivel local: la necesidad de medir los efectos", Revista Universitaria de Ciencias del Trabajo, 9: 23-39.

Cárdenas, N. (2002), “El desarrollo local su conceptualización y procesos”, Provincia, 8: $53-76$.

Carrillo, E. (2000), “Desarrollo Local. Iniciativa Pública y Empleo”, en Asociación Profesional de Formación y Empleo. Desarrollo Local y Empleo en Tiempos de Globalización. Granada: Diputación Provincial de Granada y CajaGranada.

Castells, M. (1996), La era de la información. (Vol. 1) La sociedad red. Madrid: Alianza.

Castro, G. (2000), "La cultura empresarial como Motor de Desarrollo Económico", Asociación Profesional de Formación y Empleo, Desarrollo Local y Empleo en Tiempos de Globalización. Granada: Diputación Provincial de Granada y CajaGranada.

Castro, J.M. (2002), "Indicadores de desarrollo sostenible urbano. Una aplicación para Andalucía”, Tesis doctoral, Universidad de Málaga. En línea: <http://www. eumed.net/tesis/jmc/index.htm> (consulta: 25 octubre 2011).

CCE (Comisión de las Comunidades Europeas) (2001), Fortalecimiento de la dimensión local de la estrategia europea de empleo. Comunicación de la Comisión al Consejo, al Parlamento Europeo, al Comité Económico y Social y al Comité de las Regiones. $\operatorname{COM}(2001) 629$ final.

168 cal para la Estrategia Europea de Empleo. COM (2000) 196. 
Cornella, A. (2004), “Cómo sobrevivir a la infoxicación”, en Curso de especialista en Gestión de la Información y del Conocimiento en el comercio, Valencia: Florida Universitaria.

Egea, A. (1999), "La asociación profesional de agentes de desarrollo local”, en Comunicaciones del curso Estrategias para la promoción económica y el empleo. Valencia: Universidad Internacional Menéndez Pelayo.

Esparcia, J. (2009), "La formación para el desarrollo local. Reflexiones desde el perfil profesional y las ofertas formativas en desarrollo local”, en J.L. García Rodríguez, ed., La organización territorial del Desarrollo Local en España (y algunos ejemplos iberoamericanos). Madrid: Asociación de Geógrafos Españoles y Universidad de La Laguna.

Ferri Durá, J. (2002), Voz "Políticas públicas”, en R. Reyes, dir., Diccionario Crítico de Ciencias Sociales. Madrid: Universidad Complutense de Madrid. En línea: <http:// theoria.org/diccionario/P/d-ferri1.htm> (consulta: 25 octubre 2011).

FORCEM (2000), Estudio de necesidades de formación de Agentes de Desarrollo Localy figuras profesionales afines. Madrid: FORCEM-CCOO.

García Rodríguez, J.L. (2009), “Introducción a la obra”, en J.L. García Rodríguez, ed., La organización territorial del Desarrollo Local en España (y algunos ejemplos iberoamericanos). Madrid: Asociación de Geógrafos Españoles y Universidad de La Laguna.

Greffe, X. (1990), Descentralizar a favor del empleo. Las iniciativas locales de desarrollo. Madrid: Ministerio de Trabajo y Seguridad Social.

Gusfield, J. (1994), “La reflexividad de los movimientos sociales: revisión de las teorías sobre la sociedad de masas y el comportamiento colectivo”, en E. Laraña y J. Gusfield, eds., Los nuevos movimientos sociales. De la ideología a la identidad. Madrid: CIS.

Ibáñez, J. (1994), El regreso del sujeto. La investigación social de segundo orden. Madrid: Siglo XXI.

Hernando, M. (1999), "Experiencias en desarrollo", en Comunicaciones del curso Estrategias para la promoción económica y el empleo. Valencia: Universidad Internacional Menéndez Pelayo.

ILPES (2001), El uso de indicadores socioeconómicos en la formulación y evaluación de proyectos sociales-Aplicación Metodológica-. Santiago de Chile: Instituto Latinoamericano y del Caribe de Planificación Económica y Social - ILPES y CEPAL.

Iniciativas para el Desarrollo Económico y Social (2003), La industria de la Madera y el Mueble en L'Horta Sud. Valencia: Mancomunidad Intermunicipal de L'Horta Sud.

Lamo de Espinosa, E. (2005), “¿Para qué la Ciencia Social? ¿Para quién escribimos?”, Nómadas, Revista Crítica de Ciencias Sociales y Jurídicas, 11. En línea: <http:// www.ucm.es/info/eurotheo/semana_ciencia/e_lamo.htm> (consulta: 25 octubre 2011).

Laraña, E. (1999), La construcción de los movimientos sociales. Madrid: Alianza.

León, J.J., Porras, M.D. y Carmona, C. (2006), “ARGOS, entra y verás. Sistema de prospección permanente del mercado de trabajo en Andalucía”, en TECNIMAP, Comunicaciones de las IX Jornadas sobre Tecnologías de la Información para la Modernización de las Administraciones Públicas. Sevilla: Instituto Nacional de Administración 
Pública. En línea: <http://administracionelectronica.gob.es/?_nfpb=true\&_pageLa bel=P3401382191279709374745\&langPae=es $>$ (consulta: 25 de octubre).

Madoery, O. (2001), "El valor de la política de desarrollo local”, en A. Vázquez Barquero y O. Madoery, eds., Transformaciones globales, instituciones y políticas de desarrollo local. Rosario: Homosapiens Ediciones.

MAP (2000), Libro Blanco para la mejora de los servicios públicos. Madrid: Ministerio de Administraciones Públicas.

Martín Criado, E. (1998), “Los decires y los haceres”, Papers, 56: 57-71.

Melucci, A. (1994), “¿Qué hay de nuevo en los nuevos movimientos sociales?”, en E. Laraña y J. Gusfield, eds., Los nuevos movimientos sociales. De la ideología a la identidad. Madrid: CIS.

Moltó, E.A. y Hernández, M. (2002), “Desarrollo local, geografía y análisis territorial integrado: algunos ejemplos aplicados”, Investigaciones Geográficas, 27: 175-190.

Morales, F. (1997), "Sistema de Indicadores Regionales (SIR). Manual de Diagnóstico Económico Municipal”, Red de Desarrollo Económico Local, Chile. En línea: < http://moraleseconoia.zxq.net/blog_de_moraleseconomia/SIR_Manual.pdf> (consulta 25 octubre 2011).

Muiños, B. y Cortegiano, G., (1998), “Desarrollo a escala local: los nuevos yacimientos de empleo", Biblio 3W. Revista Bibliográfica de Geografía y Ciencias Sociales, 113. En línea: <http://www.raco.cat/index.php/biblio3w/article/view/65546/75769> (consulta: 25 octubre 2011).

Navarro, P. (1989), "Cybernetics: from science of control to control of science", en J. Ibáñez, coord., Nuevos avances en la investigación social (I). Barcelona: Anthropos Editorial.

Otero, M. y Serra, J. (1998), "Regió metropolitana de Barcelona. Indicadors municipals", Papers, 30: 9-19.

Pérez-Agote, A. y Tejerina, B. (1990), “Lengua y actor social. Un enfoque teórico de sus relaciones", Revista Española de Investigaciones Sociológicas, 49: 145-160.

Pérez, B. (2000a), “Estrategias y Modelos de Desarrollo Local”, en Asociación Profesional de Formación y Empleo, Desarrollo Local y Empleo en Tiempos de Globalización. Granada: Diputación Provincial de Granada y CajaGranada.

Pérez, B. (200ob), “Las Administraciones Públicas y el Desarrollo Local”, en B. Pérez Ramírez y E. Carrillo Benito, coords., Desarrollo Local: Manual de Uso. Madrid: ESIC-FAMP.

Pérez, B. y Carrillo, B. (2000), “El Modelo Teórico del Desarrollo Local”, en B. Pérez Ramírez y E. Carrillo Benito, coords., Desarrollo Local: Manual de Uso. Madrid: ESIC-FAMP.

PNUD (2000), Informe sobre Desarrollo Humano 2000. Madrid: Mundi-prensa, En línea: <http://hdr.undp.org/es/informes/mundial/idh2000> (consulta: 25 octubre 2011).

Ramírez, L.M. (2003), El tejido empresarial del territorio, UTEDLT Sierra Oriental, Huelva. Sevilla: Unidades Territoriales de Empleo, Desarrollo Local y Tecnológico de Andalucía.

Rivera, T. (2003), Análisis demográfico l. Estructura territorial de la población y dinámica reciente del ámbito UTEDLT Sierra Oriental. Sevilla: Unidades Territoriales de 170 Empleo, Desarrollo Local y Tecnológico de Andalucía. 
Rubio, R. (2000), "Globalización y mercado de trabajo: retos y oportunidades para la promoción del empleo en el medio local”, Scripta Nova. Revista Electrónica de Geografía y Ciencias Sociales, 69(60), En línea: <http://www.raco.cat/index.php/ scriptanova/article/view/58811/68931> (consulta: 25 octubre 2011).

Sánchez, S. (2000), "Movilización social: aspectos prácticos”, Curso de Formación para Agentes de Inserción Socio-laboral (vol. I). Valencia: Universidad Nacional de Educación a Distancia.

Sanchis, J.R. (2000a), "Desarrollo local y fomento de empleo", Curso de Formación para Agentes de Inserción Socio-laboral (vol. I). Valencia: Universidad Nacional de Educación a Distancia.

Sanchis, J.R. (2000b), “Habilidades empresariales para el desarrollo”, Curso de Formación para Agentes de Inserción Socio-laboral (vol. II). Valencia: Universidad Nacional de Educación a Distancia.

Sanchis, J.R. (2005), “El papel del agente de empleo y desarrollo local en la implementación de las políticas locales de empleo y en la creación de empresas en España. Estudio empírico y análisis comparativo entre CC.AA". Informes y Estudios Empleo, 26. Madrid: Ministerio de Trabajo y Asuntos Sociales.

Sanchis, J.R. et al. (2001), "El Agente de Fomento en el proceso de Desarrollo local $y$, en especial, en la creación y desarrollo de empresas: un estudio empírico aplicado al conjunto del Estado español", Informe final del proyecto de Becas de ayuda a la investigación de la UNED. Madrid: Universidad de Educación a Distancia.

SDLPC (2009), Estudio socioeconómico de Catarroja 2009. Valencia: Ayuntamiento de Catarroja.

Servicio Andaluz de Empleo (2003), Estudio Nivel de Pervivencia de autónomos y autónomas de la Comarca Consorcio Vega Media, Sevilla: Unidades Territoriales de Empleo, Desarrollo Local y Tecnológico de Andalucía.

Sevilla, M.A. (2006), "Utilización de los registros administrativos con fines estadísticos. Una experiencia en la Seguridad Social (muestra anónima continua de vidas laborales)", en TECNIMAP, Comunicaciones de las IX Jornadas sobre Tecnologías de la Información para la Modernización de las Administraciones Públicas. Sevilla: Instituto Nacional de Administración Pública. En línea: <http://administracionelectronica.gob.es/?_nfpb=true\&_pageLabel=P3401382191279709374745\&langP ae=es $>$ (consulta: 25 de octubre).

Tejerina, B. (1992), Nacionalismo y lengua. Madrid: CIS y Siglo XXI.

Therborn, G. (1987), La ideología del poder y el poder de la ideología. Madrid: Siglo XXI. UTEDLT (2002), Indicadores básicos del territorio. Sevilla: Junta de Andalucía.

Valdivielso, R. (2002), Voz "Políticas públicas (análisis de)", en R. Reyes, dir., Diccionario Crítico de Ciencias Sociales. Madrid: Universidad Complutense de Madrid. En línea: <http://theoria.org/diccionario/P/d-valdivielso2.htm> (consulta: 25 octubre 2011).

Vázquez Barquero, A. (2000a), "La política de Desarrollo Local en Tiempos de Globalización”, en Asociación Profesional de Formación y Empleo. Desarrollo Local y Empleo en Tiempos de Globalización. Granada: Diputación Provincial de Granada y CajaGranada. 
Vázquez Barquero, A. (200ob), “Desarrollo Local y Territorio”, en B. Pérez Ramírez y E. Carrillo Benito, coords., Desarrollo Local: Manual de Uso. Madrid: ESIC-FAMP.

Vázquez Barquero, A. (1999), Desarrollo, redes e innovación. Lecciones sobre desarroIlo endógeno. Madrid: Pirámide.

Vázquez Barquero, A. (1993), Política Económica Local. Madrid: Pirámide.

Vázquez Barquero, A. (1988), Desarrollo Local. Una estrategia de creación de empleo. Madrid: Pirámide.

Wilson, F. (2005), "Información Comunal para Diagnósticos y Análisis de Proyección Socioeconómica”, Red de Desarrollo Económico Local, Chile. En línea: <http://cdi. mecon.gov.ar/biblio/docelec/MU2084.pdf> (consulta: 25 octubre 2011). 